\title{
Electron density measurement under the influence of auroral precipitation and electron beam injection during the DELTA campaign
}

\author{
M. Wakabayashi and T. Ono \\ Planetary Plasma Physics Laboratory, Graduate School of Science, Tohoku University, Sendai 980-8578, Japan
}

(Received November 15, 2005; Revised June 26, 2006; Accepted July 3, 2006; Online published September 29, 2006)

\begin{abstract}
The direct observation of electron number density was successfully carried out using an impedance probe onboard the S310-35 sounding rocket during the DELTA (Dynamics and Energetics of the Lower Thermosphere in Aurora) campaign, which provided the altitude profile along the rocket trajectory in a diffuse aurora. The plasma density profile showed clear density enhancements due to the auroral precipitation as well as artificial ionizations induced by the electron beam injection. The enhanced ionization due to the electron beam experiment was too high to be determined by means of the detection of the UHR (Upper Hybrid Resonance) frequencies. To overcome this difficulty, SHR (Sheath Resonance) frequency and sheath capacitance values were used to deduce the plasma density in the artificially ionized region. Based on the SHR analysis method, we were able to determine the maximum density to be $2 \cdot 10^{6} \mathrm{~cm}^{-3}$ at the altitude of $98.9 \mathrm{~km}$; this measurement was ascribed to the artificial ionization by the $1-\mathrm{keV}$ electron beam in comparison with the results of particle detector instrument on-board the S310-35. The data analysis of the sheath capacitance was able to distinguish the natural ionization region from the artificial one. The impedance probe measurement was able to evaluate the ionospheric conductivities which have important properties for evaluating the Joule heating process in the thermosphere. Both Pedersen and Hall conductivities showed distinctive enhancement around an altitude of $128 \mathrm{~km}$ which were due to the auroral particle precipitation.
\end{abstract}

Key words: Impedance probe, electron density, auroral precipitation, electron beam injection, polar ionosphere.

\section{Introduction}

Due to the precipitation of auroral electrons and ions, the polar ionosphere has been recognized as a complex medium of plasmas and neutral gasses in terms of energetics, physical and chemical processes. In this region, ionization and atmosphere heating due to collisions, chemical reactions, Joule heating, tide and gravity waves significantly control the energetics and dynamics of the environment. Among the elementary processes, atmosphere heating due to auroral precipitation and Joule heating control the majority of interactions between neutral gasses and plasmas of the polar upper atmosphere. Atmospheric heating resulted in strong vertical and horizontal neutral winds being observed by FPI (Fabry-Perot Interferometer) in previous studies (e.g., Price et al., 1995). The series of ARIA (Atmospheric Response in Aurora) rocket campaigns which focused on the relation between energy input and modification of the neutral winds in diffuse aurora was carried out at Poker Flat in 1992-1994 (e.g., Brinkman et al., 1995). Larsen et al. (1995) reported that neutral wind observed using the TMA (trimethyl aluminum) release experiment revealed strong shear structure near the altitude of $115 \mathrm{~km}$. They examined the generation mechanism of the observed shear structure during unstable conditions (observed Richardson Number $R_{i}$ is $<0.25$ ). However, their model calculations did not re-

Copyright (c) The Society of Geomagnetism and Earth, Planetary and Space Sciences (SGEPSS); The Seismological Society of Japan; The Volcanological Society of Japan; The Geodetic Society of Japan; The Japanese Society for Planetary Sciences; TERRAPUB produce the structure. Parish et al. (2003) carried out 3-D high-resolution computer simulations for the condition of the ARIA campaign; however, almost no wind shear structures appeared in the simulation results. To explain this contradiction, an additional energy source is suggested to drive the strong neutral wind and to maintain the shear structure. To understand the observational fact, further quantitative evaluation of atmospheric heating was needed in the polar ionosphere. Auroral precipitation itself causes direct ionospheric heating; on the other hand, ionospheric currents cause indirect heating. Odom et al. (1997) estimated the atmospheric conductivity by using the in-situ electron density data during ARIA II. They pointed out that the fieldaligned current played a significant role as the source of atmospheric heating and driving force of neutral winds. To evaluate the atmosphere heating due to the ionosphere currents, reliable measurement of the ionospheric conductivities became important. However, the measured plasma density has an ambiguity because of the usage of the Langmuir probe technique.

The DELTA (Dynamics and Energetics of the Lower Thermosphere in Aurora) campaign aimed to obtain accurate heating rates and to quantitatively compare these with the atmosphere temperature and wind. To realize the accurate measurement of the neutral gas temperature, the electron beam fluorescence method has been developed onboard a sounding rocket (Kurihara, 2003). This instrument provided an important advantage in the present experiment DELTA (as described by Kurihara et al., 2006). 
The DELTA campaign consisted of the S310-35 sounding rocket experiment and ground-based observations such as FPIs, all sky cameras and the EISCAT (European Incoherent Scatter) radar [Abe et al. (2006) introduced the scientific aim of the DELTA and all the instruments on-board the rocket]. The electron density measurement using the impedance probe technique was carried out on-board the sounding rocket simultaneously with the electron beam experiment (detailed descriptions are in Section 2 and 3.1). The impedance probe technique has an important advantage in that is able to identify the absolute density value by detecting the Upper Hybrid Resonance (UHR) frequency of ambient plasma. In addition, it is also able to measure the Sheath Resonance (SHR) frequency as well as the sheath capacitance $\left(C_{s}\right)$. The SHR frequency and the $C_{s}$ value are also useful for deriving the electron density.

Because the electron beam experiment is expected to have a significant effect on the ambient plasma around the rocket body, it is important to clarify the artificial effects on the observed plasma parameters.

This paper reports the results of the electron density measurements, with evaluating the artificial effects due to the electron beam experiment, and the ionospheric conductivities deduced from the electron density profile of the ambient plasma.

\section{On-board Instrumentation for the DELTA Campaign}

The impedance probe instrument called NEI (Number density of Electrons by Impedance probe) was installed in the $\mathrm{S} 310-35$ rocket to measure the absolute electron density along the rocket trajectory. This instrument can determine the UHR frequency of the ambient plasma by measuring an equivalent capacitance of the conductor probe in space plasma (Oya, 1966). NEI measurement also provides the SHR frequency and $C_{s}$. Figure 1 shows a schematic relation of the probe, rocket body and ambient plasma. In Fig. 1, the equivalent capacitance $C(\omega)$ of the resonant circuit is obtained as

$$
C(\omega)=\frac{1}{|Z(\omega)|}=\left(\frac{1}{\omega C_{s}}+\frac{1}{\omega C_{a}-1 / \omega L_{p}}\right)^{-1},
$$

where, $Z(\omega)$ and $\omega$ are the impedance of the resonant circuit and angular frequency of RF signals, respectively. The equivalent capacitance has maximum and minimum values when $\omega$ is

$$
\omega_{\mathrm{SHR}}=\frac{1}{\sqrt{L_{p}\left(C_{a}+C_{s}\right)}}
$$

(a series resonance: corresponds to SHR)

and

$$
\omega_{\mathrm{UHR}}=\frac{1}{\sqrt{L_{p} C_{a}}}
$$

(a parallel resonance: corresponds to UHR).

Figure 2 shows an example of the NEI data revealing the maximum (SHR) and minimum (UHR) of the equivalent capacitance. When the RF signal is applied at a very low frequency, the effect of the inductance $L_{p}$ becomes negligible. Then the sheath capacitance can be obtained at the low-

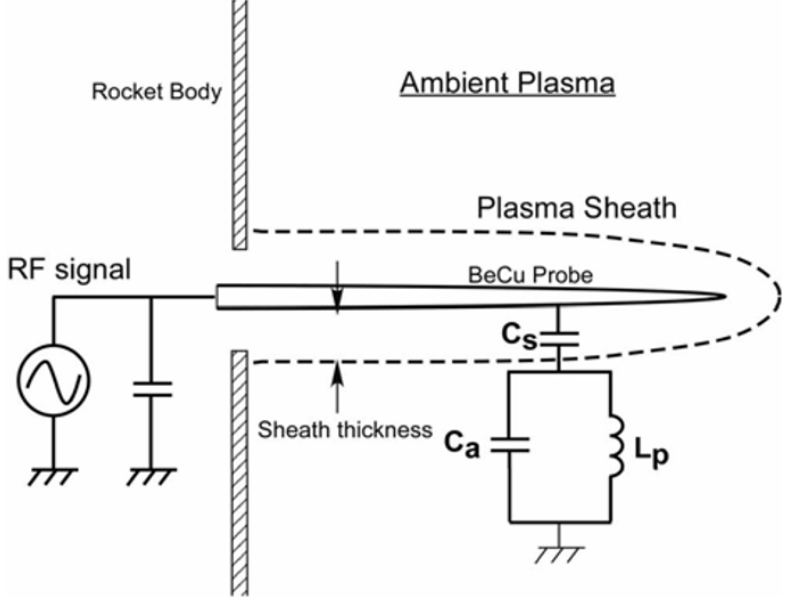

Fig. 1. A model of antenna impedance immersed in plasma for the NEI observation. $C_{s}, C_{a}$ and $L_{p}$ represent sheath capacitance, probe capacitance and plasma inductance, respectively.

13/12/2004 NEI rawdata/S310-35

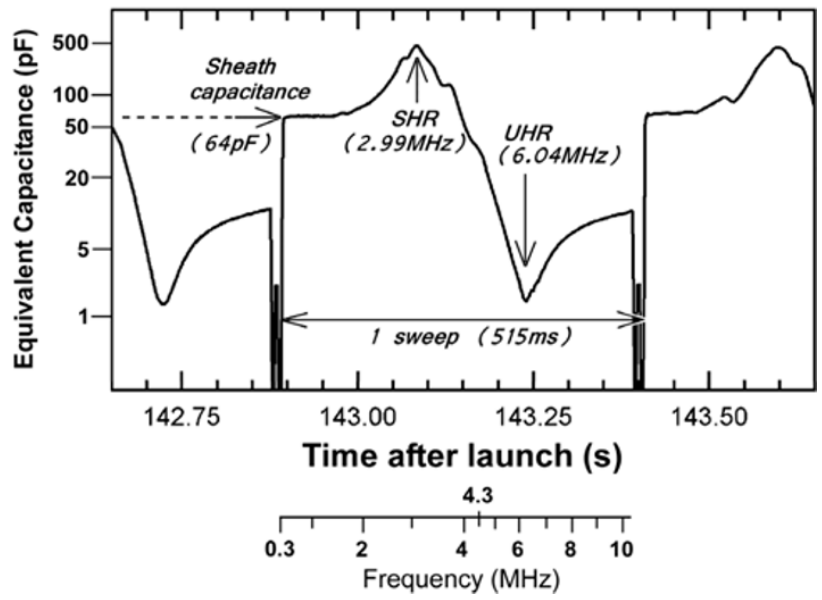

Fig. 2. An example of NEI data on-board the S310-35. As shown in this figure, we can identify the UHR and SHR frequencies as the dip and peak points of equivalent capacitance, respectively. The sheath capacitance is measured at the lowest frequency $(300 \mathrm{kHz})$.

est frequency range of $300 \mathrm{kHz}$. From the UHR frequency, we can obtain the absolute electron density with the relation

$$
N_{e}=1.24 \times 10^{4}\left(f_{\mathrm{UHR}}^{2}-f_{c}^{2}\right)
$$

where $N_{e}, f_{\mathrm{UHR}}$ and $f_{c}$ are the electron number density $\left(\mathrm{cm}^{-3}\right)$, the UHR frequency $(\mathrm{MHz})$ and the electron cyclotron frequency $(\mathrm{MHz})$, respectively. The accuracy of the absolute electron density value is basically determined by the readout error of the UHR, which depends on the frequency step of the RF signal. The measurable range of electron density is from $10^{3}$ to $1.2 \cdot 10^{6} \mathrm{~cm}^{-3}$. The time resolution of NEI is $515 \mathrm{~ms}$, which is due to the time period of the frequency sweep from $300 \mathrm{kHz}$ to $10.3 \mathrm{MHz}$. On the other hand, the SHR frequency is a function of electron density, temperature and the direction of the magnetic field. The sheath capacitance is a function of the electron density, temperature and the probe potential and can provide useful information on ambient plasma, as described by Oya and Aso (1969). To protect the capacitance bridge cir- 
cuit from the possible high-energy electron input due to the electron beam injection, the present NEI probe was connected to the ground by a $10-\mathrm{M} \Omega$ resistor, while previous NEI experiments (Wakabayashi et al., 2005, Wakabayashi and Ono, 2005) used the floating probe condition. The NEI probe consisted of a 1.2-m Copper-Beryllium ( $\mathrm{BeCu}$ alloy) ribbon antenna (diameter: $12 \mathrm{~mm}$ ). The release of an extension lock could deploy this probe immediately.

To avoid any potential changes due to electron beam injection by NTV (nitrogen temperature instrument), S310-35 was equipped with a rocket separation mechanism. In this article, the motor side and the nosecone side of the S31035 will be identified as the "mother rocket" and "daughter rocket", respectively. NEI was installed in the "mother rocket" section of the S310-35 to avoid interference from the NTV in the "daughter rocket" section. The NEI probe was deployed through a door in the skin of the "mother" rocket body. The detail configurations of the mother and daughter rockets are described in the paper of Abe et al. (2006).

\section{Observation Results}

\subsection{Flight operation}

The launch of the sounding rocket for the DELTA campaign was carried out on 13 December, 2004 at the Andøya Rocket Range $\left(69.29^{\circ} \mathrm{N}, 16.01^{\circ} \mathrm{E}\right)$ in Norway. The overview of this campaign has been described in the paper of Abe et al. (2006). The EISCAT radar, FPIs, all-sky cameras and other ground-based instruments were operated simultaneously with the rocket flight during this campaign. The sounding aim was to launch the rocket during a diffuse aurora condition because we expected that the ionosphere over Troms $\varnothing$ and Andøya to be homogeneous in that condition. This condition is valuable when wishing to evaluate the direction and intensity of the electric field in the ionosphere by means of the EISCAT radar observation over Troms $\varnothing$, which is located $130 \mathrm{~km}$ east of Andøya. The launch time of the rocket was determined to be 0:33 UT (1:33 LT) by using the result of EISCAT observation. The rocket was launched toward the azimuth of $0.45^{\circ}$ and elevation of $84.6^{\circ}$. The rocket flight was successfully carried out as planned, however, a small auroral breakup eventually occurred simultaneously with the rocket launch. As a result, the rocket passed in the auroral active region in the ascending phase while it passed in the quiet region in the descending phase. Therefore, the spatial homogeneity was reduced during this campaign.

Figure 3 shows the rocket trajectory and timer sequences of the NEI and NTV. As shown in Fig. 3, the NEI probe was extended at $65 \mathrm{~s}$ after the launch at the altitude of 72.9 $\mathrm{km}$. At $84 \mathrm{~s}$ after the launch, the heater of the electron gun's cathode was turned on. At 89 s, the high voltage (HV) was applied on the electron gun. The electron gun worked continuously from the time of HV ON except for the altitude region near the apogee (not represented in Fig. 3). The rocket was separated at $100 \mathrm{~s}$, at a $106.7 \mathrm{~km}$ altitude. All on-board instruments worked perfectly during the flight.

\subsection{UHR analysis}

The altitude profiles obtained by using the NEI instrument are shown in Figs. 4(a) and (b). Below the altitude

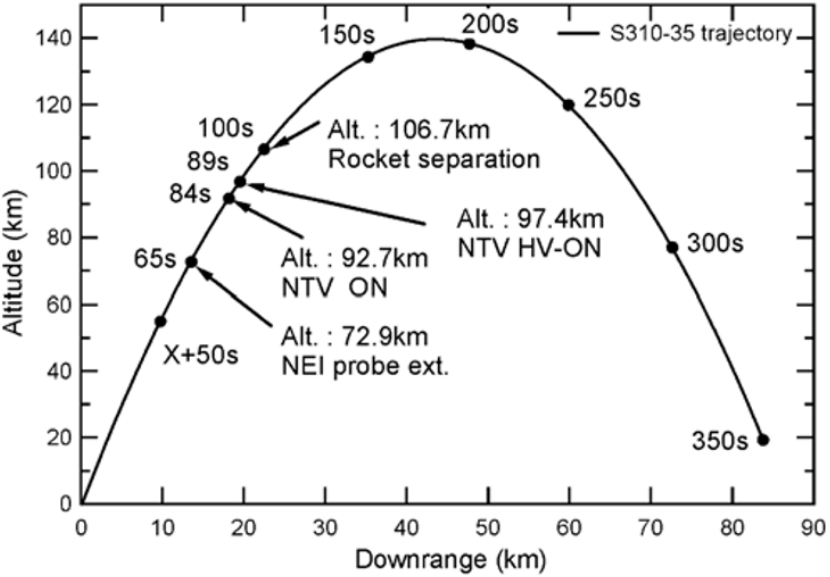

Fig. 3. Trajectory and flight events of the NEI and NTV instruments of the $\mathrm{S} 310-35$ rocket experiment.

of $98 \mathrm{~km}$, the electron density profiles measured during the ascending and descending phases show a similar tendency. It should be noted that electron-neutral collision effects below the altitude of $100 \mathrm{~km}$ have been corrected using the MSIS-E-90 atmospheric model. Above $98 \mathrm{~km}$ in each figure, there are the clear plasma density increases in the $E$ region ionosphere. During the ascending phase, there are two density enhancements in the altitude range from 104 to $112 \mathrm{~km}$, and from 124 to $132 \mathrm{~km}$. From the time of NTV$\mathrm{HV} \mathrm{ON}$ at $89 \mathrm{~s}$, the UHR frequency became too large to be identified by NEI until the adjacent time of rocket separation at $100 \mathrm{~s}$. Figure 5 represents the NEI raw data, which imply that there have been dense plasma regions larger than $1.2 \cdot 10^{6} \mathrm{~cm}^{-3}$ after the NTV-HV ON. Even though UHR detection recovered to be available again after the rocket separation, there are still some data gaps in Fig. 4(a) around the $110 \mathrm{~km}$ altitude. On the other hand, during the descending phase [in Fig. 4(b)], the density enhancement appears in the altitude range 108 to $114 \mathrm{~km}$. There are also additional small enhancements at the altitude range 129 to 140 $\mathrm{km}$. As shown in the Fig. 4(b), periodic fluctuations in electron density appear; those are due to the effect of the rocket wake.

\subsection{SHR analysis}

As shown in Fig. 4(a), there are data gaps in the electron density profile based on the detection of the UHR frequency in the ascending phase. To complete the task for the plasma density measurement of the NEI, alternative methods are worth attempting. We can apply the data analysis of the SHR frequency. When we compare the observed SHR frequency and electron density based on the determination of the UHR frequency within the up-leg observation period shown in Fig. 4(a), there is the linear relation, as shown in Fig. 6. This relationship can be described by the equation of

$$
N_{e}=\left(-3.36+2.43 \cdot f_{\mathrm{SHR}}\right) \times 10^{5},
$$

where, $N_{e}$ and $f_{\mathrm{SHR}}$ are the electron density $\left(\mathrm{cm}^{-3}\right)$ and SHR frequency $(\mathrm{MHz})$, respectively. The correlation coefficient is 0.942 , which represents the intense positive correlation.

As a first approximation, we applied this empirical rela- 
(a)

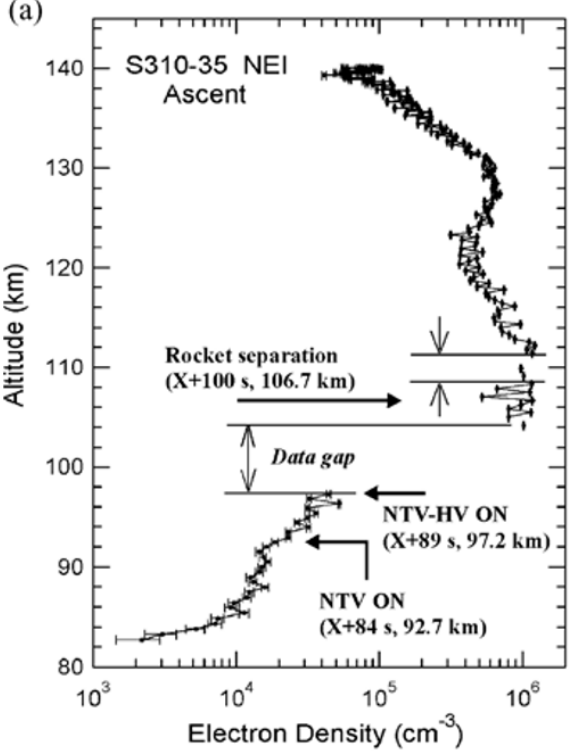

(b)

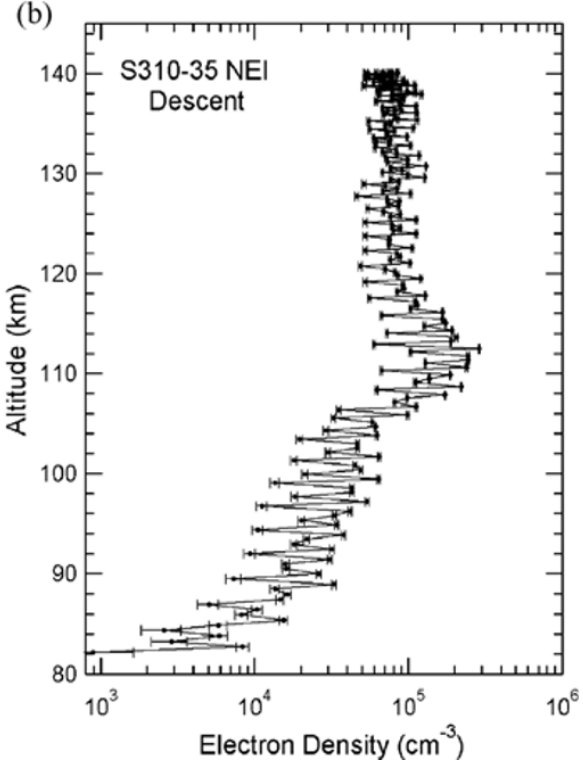

Fig. 4. (a) and (b). Electron density profiles obtained by using the NEI instrument based on the UHR method. (a) and (b) panels show the ascent and descent, respectively.

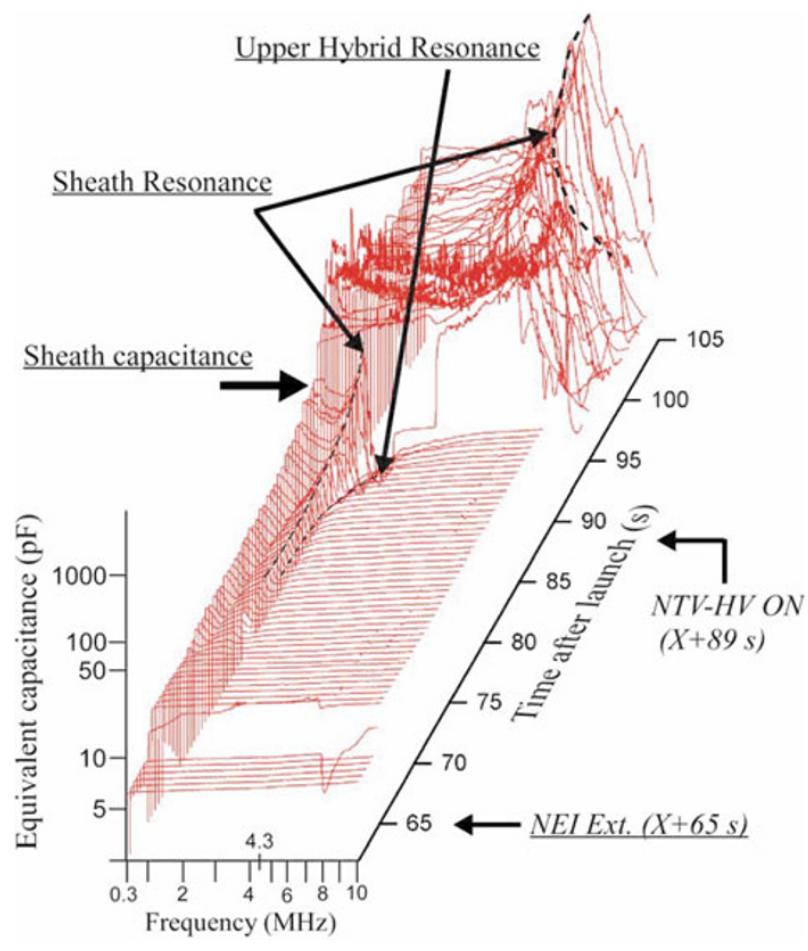

Fig. 5. Sequence of NEI data from 60 to $105 \mathrm{sec}$ after launch. The UHR frequency moved out of the measurable range after the NTV-HV ON.

tion between the density and SHR frequency, although the SHR frequency not only depends on the electron density but also on other plasma parameters, such as the electron temperature and the magnetic field direction. Based on this empirical relationship, the calculated electron density is overplotted in Fig. 7. The deduced density from the SHR agrees well with the observed electron density as shown in Fig. 7. By using this method, the maximum density can be estimated as $2 \cdot 10^{6} \mathrm{~cm}^{-3}$ at an altitude of $98.9 \mathrm{~km}$. However,

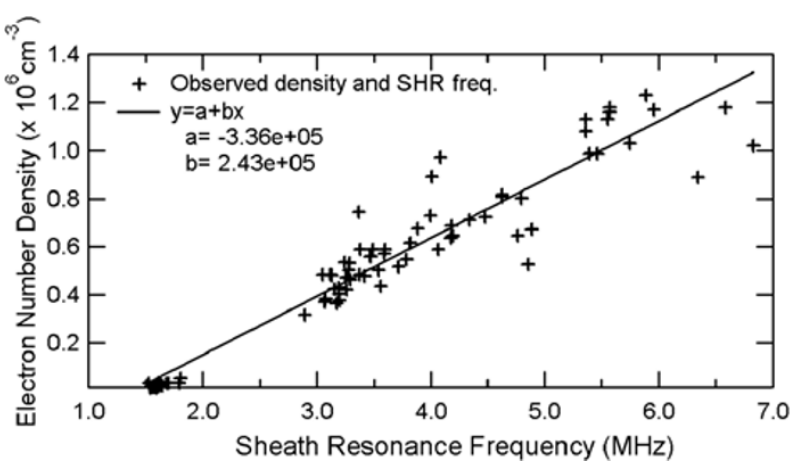

Fig. 6. Relationship between the electron density with the UHR method and the SHR frequency measured at the same time.

there are also data gap regions because the SHR frequencies also become out of the range of the NEI frequency coverage.

\subsection{Sheath analysis}

The next trial is on the analysis of the measured sheath capacitance. The basic theory of the sheath was studied by Oya and Aso (1969), and Aso (1973). The sheath capacitance $C_{s}(\mathrm{~F})$ is described as the capacitance of the cylindrical condenser as

$$
C_{s}=\frac{2 \pi \varepsilon_{0} l}{\ln \left(R_{s} / R\right)}
$$

where, $R_{s}, R, l$ and $\varepsilon_{0}$ are the sheath radius $(\mathrm{m})$, probe radius $(\mathrm{m})$, probe length $(\mathrm{m})$ and dielectric constant, respectively (Oya and Aso, 1969). We adopted $R$ and $l$ as $0.006 \mathrm{~m}$ and $1 \mathrm{~m}$, respectively. We assume that an effective sheath thickness $\left(R_{s}-R\right)$ is proportional to the Debye length described as

$$
R_{s}-R=\alpha \lambda_{D},
$$

where, $\alpha$ and $\lambda_{D}$ are constant and the Debye length, respectively. 


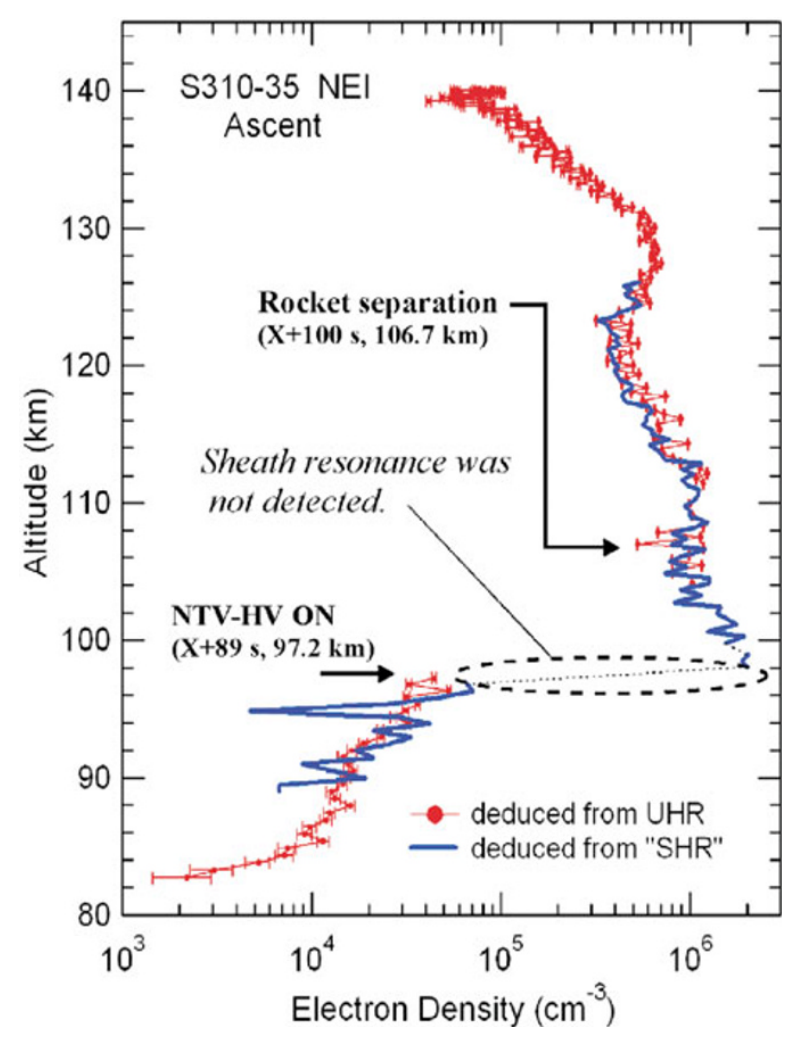

Fig. 7. Altitude profile of electron density deduced from the relation in Fig. 6.

Then, we can obtain the relation of

$$
R_{s}=\alpha\left(\frac{\varepsilon_{0} k_{B} T_{e}}{N_{e} e^{2}}\right)^{1 / 2}+R
$$

where, $k_{B}$ and $T_{e}$ are Boltzmann's constant and electron temperature $(\mathrm{K})$.

Using Eqs. (6) and (8), we can deduce the electron density as

$$
N_{e}=\frac{\alpha^{2} \varepsilon_{0} k_{B} T_{e}}{e^{2} R^{2}\left\{\exp \left(\frac{2 \pi \varepsilon_{0} l}{C_{s}}\right)-1\right\}^{2}} .
$$

In this relationship, Aso (1973) showed good agreement between rocket observation results in the mid-latitude ionosphere and theoretical calculation with adopting the factor of " 3 " for $\alpha$. We will also use this constant value of 3 in our analysis. In this analysis, electron temperature was provided by the FLP (Fast Langmuir Probe) measurement (Abe and Oyama, 2006) which is available above an altitude of $105 \mathrm{~km}$. Below an altitude of $105 \mathrm{~km}$, we extrapolated the electron temperatures above that height. The results of electron density analysis based on this method are plotted in Fig. 8 which compares the results of three methods described previously. In Fig. 8, the results by using $C_{s}$ are given by the dotted line below $105 \mathrm{~km}$. As shown in Fig. 8, results of the sheath capacitance method show disagreements with the UHR and SHR methods within the altitude range between 123 and $131 \mathrm{~km}$ and below $115 \mathrm{~km}$. The sheath capacitance shows significant fluctuations in the altitude region below $115 \mathrm{~km}$. These fluctuations start from the time of NTV-HV ON, and they gradually diminish following rocket separation.

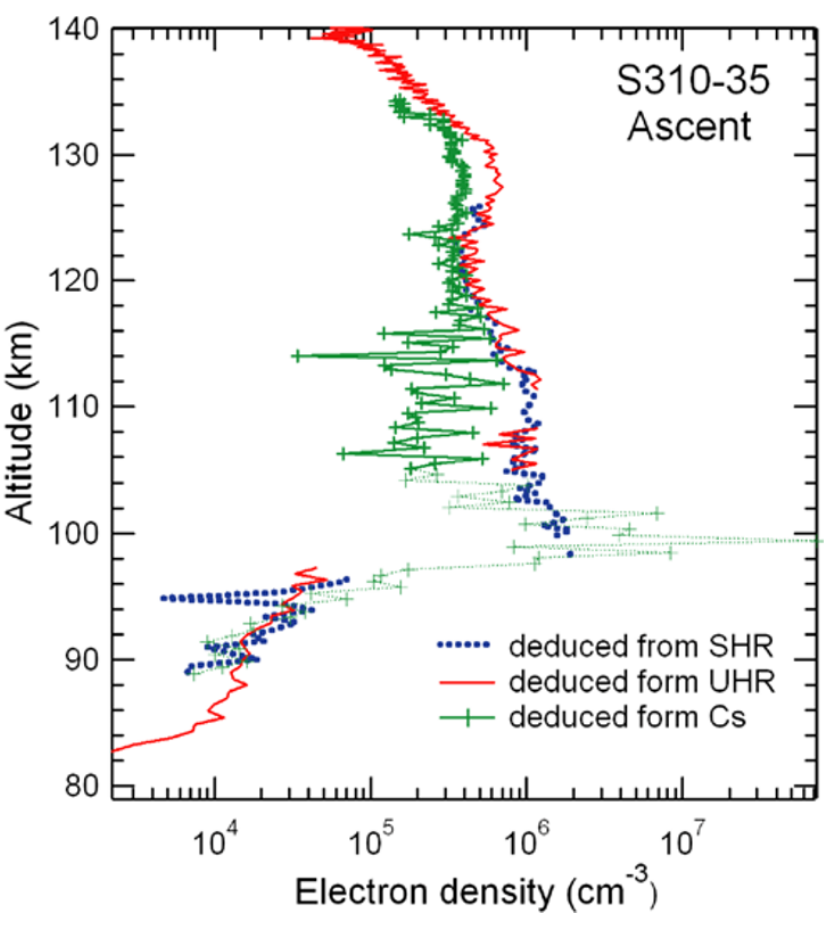

Fig. 8. Comparison between the density values deduced from the UHR, SHR frequencies and sheath capacitance.

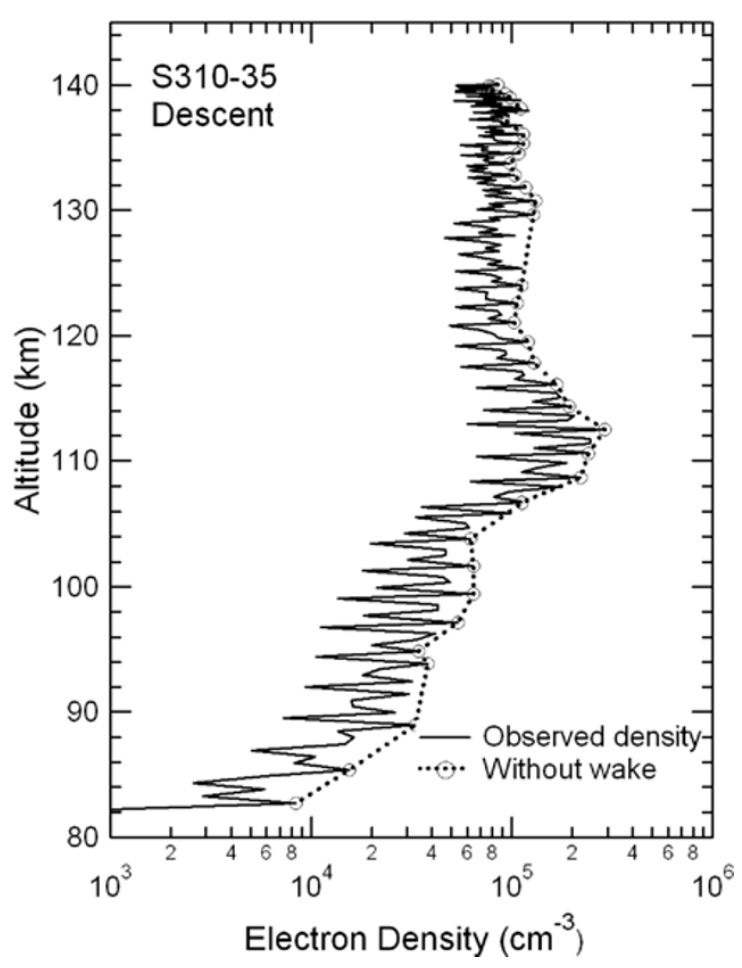

Fig. 9. Density profiles after the elimination of wake effects.

\subsection{Wake effects during the descending phase}

During the descending phase, the observed plasma density shows fluctuations according to the rocket spin due to the wake effects [see Fig. 4(b)]. When we selected the NEI data corresponding to spin phase angle within $\pm 30^{\circ}$ (namely, ram direction), which was indicated by the onboard magnetic sensor, wake effects were eliminated, as 


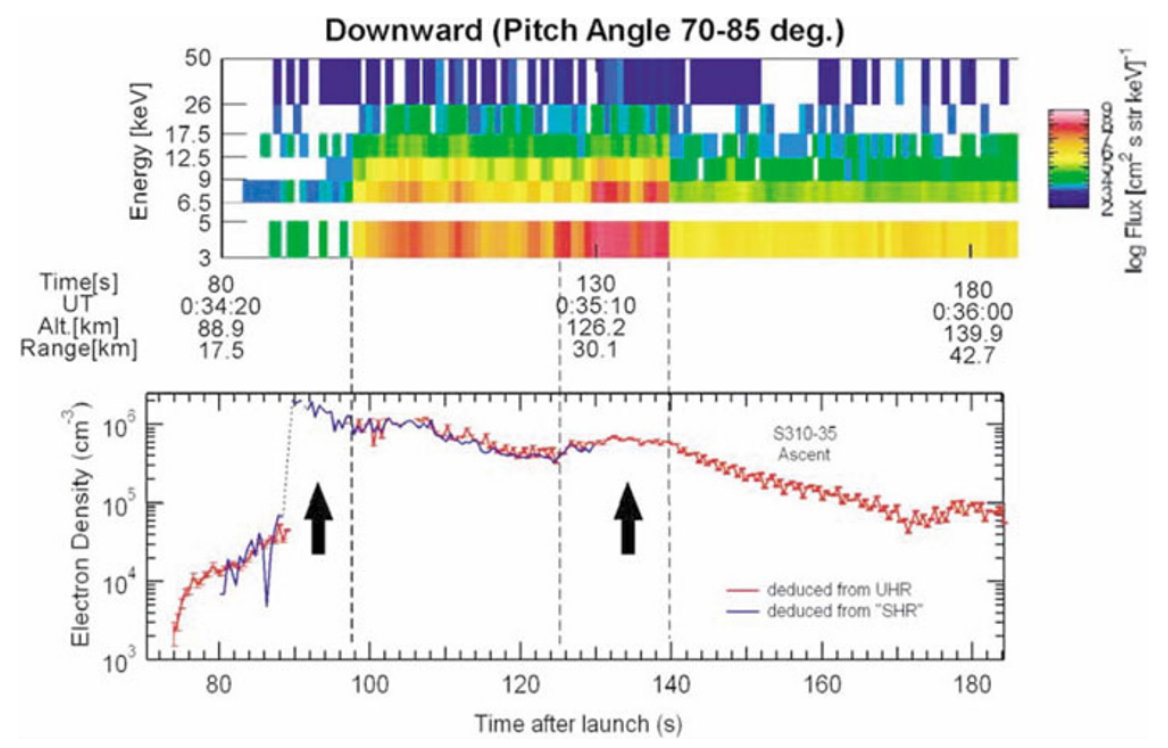

Fig. 10. Comparison between the variation of electron density and precipitating auroral electrons measured by APD instrument. The energy-time (ET) diagram was provided by Ogasawara (private communication).

shown in Fig. 9. Following wake elimination, the density profile gives the envelope of maxima of the measured density values.

\section{Discussion}

\subsection{Evaluations of electron density profiles}

We have obtained electron density values along the rocket trajectory by three analysis methods (UHR, SHR frequencies and $C_{s}$ values). The SHR analysis has shown a maximum density enhancement of $2 \cdot 10^{6} \mathrm{~cm}^{-3}$ at an altitude of $98.9 \mathrm{~km}$. This analysis method is not established theoretically; however, the validity of this analysis has been examined empirically in the observation period of NEI when the measurements of UHR frequency give accurate plasma densities.

In previous studies, electron density values often reached $10^{6} \mathrm{~cm}^{-3}$ in an active auroral arc (e.g., Takahashi et al., 1988). It was also reported that the peak altitude was correspondent to the average energy of the auroral particles. In Fig. 10, electron density is compared with the measured particles obtained by using the APD (Auroral Particle Detector) instrument (Ogasawara et al., 2006). Figure 10 shows that the highest peak of density at an altitude of $98.9 \mathrm{~km}$ does not correspond to the strong particle precipitation. Therefore, this result suggests that such a high density enhancement is not due to the auroral precipitation but, instead, to an artificial ionization effect by the NTV experiment. On the other hand, density enhancements in the altitude range from 124 to $132 \mathrm{~km}$ show good agreement with the strong precipitation of auroral particles. It would appear that the NEI have measured the ambient plasma in this region.

It is important to identify the region where the observed plasma density values are strongly affected by the artificial ionization by the NTV experiment. Electron densities deduced from the sheath capacitance values showed significant differences in two regions (in Fig. 8). The difference in the altitude range from 123 to $131 \mathrm{~km}$ is relatively small, and this can be interpreted as caused by changes in the probe potential due to the auroral particle precipitation. In this case, it seems that the velocity distribution of the plasma was non-Maxwellian. Therefore, the electron density does not agree with the value, assuming the condition of floating potential. The large difference with significant fluctuations between 105 and $115 \mathrm{~km}$ is also attributable to energetic particles [see Fig. 10]; however, the difference is relatively larger than the one in the altitude range of 123$131 \mathrm{~km}$ where auroral precipitation occurred. Therefore, this region is likely to be affected not only by potential changes but also by artificial ionization due to the NTV. On the other hand, the electron density deduced from $C_{s}$ becomes extremely large $\left(>10^{7} \mathrm{~cm}^{-3}\right)$ in the altitude range from 96 to $105 \mathrm{~km}$; this is attributable not only to the effects of the rocket potential change but also to the extrapolation of electron temperature. In the altitude region of 96-105 $\mathrm{km}$, the density values are significantly affected by electron temperature by Eq. (9) where the sheath capacitance values are about 50-60 pF. Consequently, the deduced density values are not meaningful in this region. The potential fluctuations (detected by the FLP) support these interpretations in the altitude range from 96 to $115 \mathrm{~km}$ in terms of the agreement between the potential fluctuation region and the density (deduced from the $C_{s}$ ) one. Below the $96 \mathrm{~km}$ altitude, where the sheath capacitances are about 20-30 pF, the deduced densities are not affected by electron temperature in Eq. (9), even if the extrapolated temperature is significantly different from actual temperatures. This fact may explain the good agreement among the three profiles below the altitude of $97 \mathrm{~km}$ in Fig. 8.

We can therefore conclude that the altitude regions below $97 \mathrm{~km}$ (before NTV-HV ON) and above $115 \mathrm{~km}$ in the ascending phase are not influenced by the artificial ionization. In the descending phase, all of the density values in Fig. 9 are due to natural phenomena because the wake effects are eliminated. 

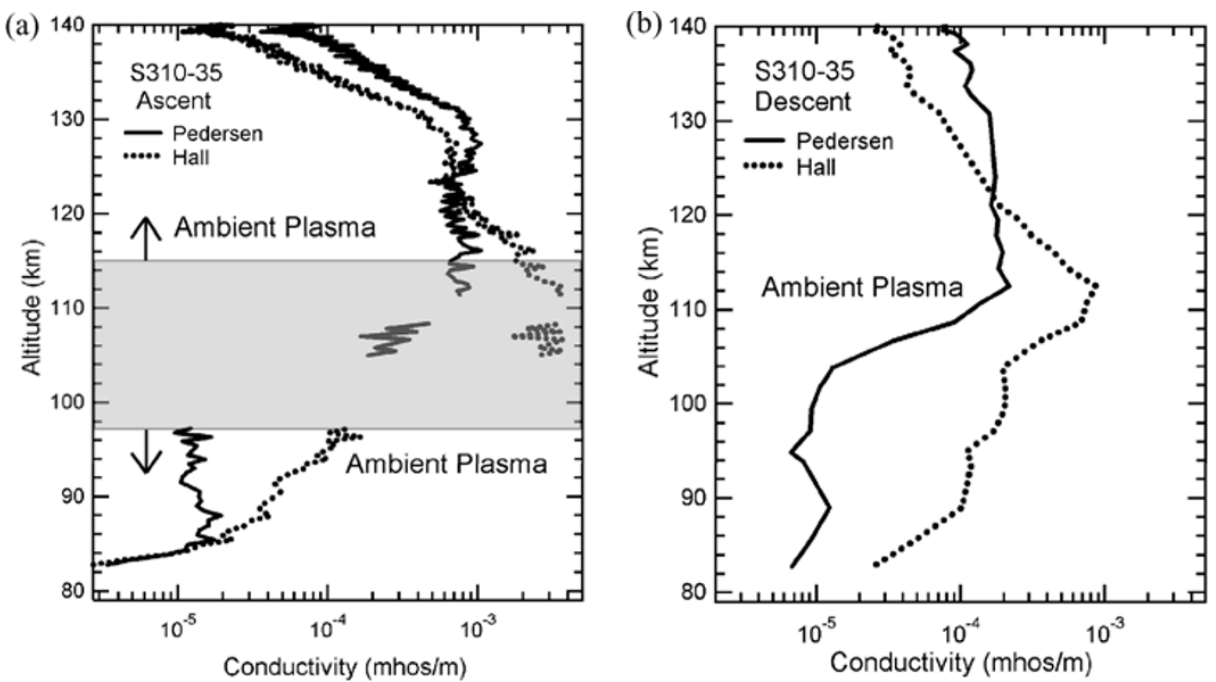

Fig. 11. (a) and (b). Atmospheric conductivity along the rocket trajectory by using the results of the NEI observation. Panels indicated as (a) and (b) show the ascent and descent, respectively. In the panel (a), gray shaded region shows that the measurement is affected by an artificial ionization. The annotation of "Ambient Plasma" indicates the altitude regions where no artificial ionization effects exist.

4.2 Comparison with the EISCAT radar observations

The EISCAT observation was used as the launch criterion during this campaign. The electron density obtained by EISCAT shows good agreement among the different beams [the EISCAT data was provided by Nozawa (private communications)]. However, the EISCAT observation shows maximum density of about $5 \cdot 10^{5} \mathrm{~cm}^{-3}$ near the altitude of $120 \mathrm{~km}(0: 31 \mathrm{UT}$ and 0:36 UT of East1 and Vertical, respectively). This point is significantly different from the rocket observation during the ascending phase, which shows the maximum density about $2 \cdot 10^{6} \mathrm{~cm}^{-3}$ at the altitude of 98.9 $\mathrm{km}$ and local enhancement at the altitude of 124-132 km. The difference between the EISCAT result and that of the rocket is due to the small auroral breakup and influence of the artificial ionization by NTV (as described in Sections 3 and 4.1). On the other hand, there is good agreement between the EISCAT and rocket data during the descending phase even though the location of the descent region is more distant from Troms $\emptyset$ than the ascent region. The rocket observation shows the maximum density of $3 \cdot 10^{5} \mathrm{~cm}^{-3}$ at the altitude of $108-114 \mathrm{~km}$ during the descending phase. This feature agrees well with the EISCAT data obtained at 0:27 UT (East2), 0:30 UT (Vertical), 0:35 UT (Field-aligned) and 0:42 UT (Vertical).

\subsection{Atmospheric conductivity}

The atmospheric conductivities can be deduced by using the electron density profiles. The Pedersen and Hall conductivities are derived as functions of cyclotron and plasma frequencies, and collision frequencies of ion-neutral $\left(v_{\text {in }}\right)$ and electron-neutral $\left(v_{\mathrm{en}}\right)$ pairs. The collision frequencies are given as the following equations (Rees and Walker, 1968; Jones, 1974),

$$
\begin{aligned}
v_{\text {in }}= & 8 \times 10^{-15}\left(\frac{4}{3}\right) \frac{\mu_{\text {in }}}{m_{i}} N_{n}\left(\frac{8 k_{B}}{\pi}\right)^{1 / 2} \\
& \times\left(\frac{T_{i}}{m_{i}}+\frac{T_{n}}{m_{n}}\right)^{1 / 2}, \\
v_{\text {en }}= & 5 \times 10^{-10} N_{n}\left(T_{e}\right)^{1 / 2},
\end{aligned}
$$

where, $m_{i}, m_{n}$ and $\mu_{\text {in }}$ are mass of the ion $(\mathrm{kg})$, mass of the neutral particle $(\mathrm{kg})$ and reduced mass of the ion and neutral particle $(\mathrm{kg})$, respectively. The $N_{n}, k_{B}, T_{i}$ and $T_{n}$ are number density of neutral particles $\left(\mathrm{cm}^{-3}\right)$, Boltzmann's constant and ion and neutral temperature $(\mathrm{K})$, respectively. The $m_{i}$ and $m_{n}$ are assumed to be the mass of $\mathrm{NO}^{+}$and the average mass of the neutral particles, respectively. $N_{n}$ is obtained from the MSIS-E-90 model. By referring to EISCAT radar data, $T_{i}$ is assumed to be $900 \mathrm{~K}$. The $T_{n}$ values have been measured using the NTV instrument onboard the rocket.

Figures 11(a) and (b) show the atmospheric conductivities which are calculated along the rocket trajectory (In Fig. 11(a), the altitude region affected by NTV was indicated by gray shading. The annotation of "ambient plasma" refers to the region not affected because NTV was far enough away or turned off). In this calculation, the electron density values and magnetic field intensity are given by the NEI data and International Geophysical Reference Field (IGRF) model, respectively. During the ascending phase, the Hall conductivity is largely enhanced below an altitude of $124 \mathrm{~km}$. Above $124 \mathrm{~km}$, the Pedersen conductivity is predominant rather than Hall conductivities. This tendency can be also seen in the descending phase. However, in the altitude region between 124 and $132 \mathrm{~km}$ in the ascending phase, there are enhancements in both conductivities. In the descending phase, there are also enhancements of both conductivities in the altitude range from 108 to 114 $\mathrm{km}$.

In comparison with previous observations in diffuse aurora by Thiele et al. (1981) (based on in-situ rocket observation), conductivity values during the DELTA are relatively large, especially during the ascending phase. Kirkwood et al. (1988) deduced the conductivity in diffuse aurora in the growth phase, expansion phase, discrete arc and westward traveling surge which were based on EISCAT observations. The conductivity enhancements in the 98-115 $\mathrm{km}$ altitude range during the ascending phase are similar to 
those obtained during the westward traveling surge reported by Kirkwood et al. (1988); however, enhancements of the present experiment are identified as artificial. On the other hand, the conductivity enhancement in the altitude range from 124 to $132 \mathrm{~km}$ seems to be the unique feature due to the auroral precipitation during the DELTA rocket experiment. It is possible to expect that there is possible atmospheric heating effect due to the auroral precipitation in the altitude range from 124 to $132 \mathrm{~km}$. During the descending phase, Pedersen conductivities are comparable to the previous observations which have been cited above.

\section{Conclusions}

A direct electron density measurement was carried out during the DELTA campaign by using the NEI instrument. The result of this NEI observation showed significant density enhancements due to the natural aurora and artificial ionization effects. Moreover, there were data gaps where the electron density was too high to be detected by the NEI. Not only to fill the data gaps but also to distinguish the natural ionization region from artificial ones, we analyzed details of the NEI data, including the variation of SHR frequency as well as the sheath capacitance. The results from SHR analysis suggested that there was a maximum electron density of $2 \cdot 10^{6} \mathrm{~cm}^{-3}$ at an altitude of $98.9 \mathrm{~km}$, which was due to artificial ionizations. The results from $C_{s}$ analysis indicated that this artificial ionization region covered the altitude range from 97 to $115 \mathrm{~km}$ in the ascending phase. The enhancement of electron density in the altitude range from 124 to $132 \mathrm{~km}$ is natural ionization due to auroral precipitation. The EISCAT data did not show good agreements with rocket results during the ascending phase. In the descending phase, observed densities were largely affected by the rocket wake. Such effects were eliminated by referring to the spin phase angle data during the flight. After eliminating the wake effect, the electron density profile during the descending phase was regarded as the profile of auroral ionosphere. The electron density profile during the descending phase showed good agreement with the EISCAT observation results. The atmospheric conductivities which were deduced from the present observation showed significant conductivity enhancements due to the aurora in the altitude range from 124 to $132 \mathrm{~km}$. These conductivity values may contribute an evaluation of the effects of Joule heating during the DELTA campaign.

Acknowledgments. The S310-35 rocket experiment was conducted by the Institute of Space and Astronautical Science/Japan Aerospace Exploration Agency (ISAS/JAXA). The authors would like to express their sincere thanks to Dr. T. Abe, Dr. H. Hayakawa and rocket team members of ISAS/JAXA; to Dr. M. Iizima, Dr. A. Kumamoto and Dr. H. Fujiwara of Tohoku University for their important discussions and comments on the present study; to Dr. J. Kurihara of ISAS/JAXA, Dr. S. Nozawa of Nagoya University, Dr. N. Iwagami and Dr. K. Ogasawara of University of Tokyo for their cooperation in carrying out the DELTA campaign and for providing the data of the sounding rockets and EISCAT radar. The authors wish to express their deep appreciation to the members of System Keisoku Inc. for their development of the rocket-borne instrument. The research activities of the authors are supported by the 21st century COE program "Advanced Science and Technol- ogy Center for the Dynamic Earth” at Tohoku University.

\section{References}

Abe, T., K. Oyama, and A. Kadohata, Electron temperature vatiation associated with the auroral energy input during the DELTA campaign, Earth Planets Space, 58, this issue, 1139-1146, 2006.

Abe, T., J. Kurihara, N. Iwagami, S. Nozawa, Y. Ogawa, R. Fujii, H. Hayakawa, and K. Oyama, Dynamics and Energetics of the Lower Thermosphere in Aurora (DELTA) - Japanese sounding rocket campaign, Earth Planets Space, 58, this issue, 1165-1171, 2006.

Aso, T., A sheath resonance observed by a high frequency impedance probe, J. Geomag. Geoelectr., 25, 325-330, 1973.

Brinkman, D. G., R. L. Walterscheid, L. R. Lyons, D. C. Kayser, and A. B. Christensen, E region neutral winds in the postmidnight diffuse aurora during the Atmospheric Response in Aurora 1, J. Geophys. Res., 100(A9), 17,309-17,320, 1995.

Jones, A. V., Aurora, D. Reidel, Hingham, Mass, 1974.

Kirkwood, S., H. Opgenoorth, and J. S. Murphree, Ionospheric conductivities, electric fields and currents associated with auroral substorms measured by the EISCAT radar, Planet. Space Sci., 36, 12, 1359-1380, 1988.

Kurihara, J., Energetics and structure of the lower thermosphere observed by sounding rocket experiment, Ph.D thesis, University of Tokyo, Japan, 2003

Kurihara, J., T. Abe, K. Oyama, E. Griffin, M. Kosch, A. Aruliah, K. Kauristie, Y. Ogawa, S. Komada, and N. Iwagami, Observations of the lower thermospheric neutral temperature and density in the DELTA campaign, Earth Planets Space, 58, this issue, 1123-1130, 2006.

Larsen, M. F., T. R. Marshall, I. S. Mikkelsen, B. A. Emery, A. Christensen, D. Kayser, J. Hecht, L. Lyons, and R. Walterscheid, Atmospheric response in Aurora experiment: observations of $\mathrm{E}$ and $\mathrm{F}$ region neutral winds in a region of postmidnight diffuse aurora, J. Geophys. Res., 100(A9), 17,299-17,308, 1995.

Odom, C. D., M. F. Larsen, A. B. Christensen, P. C. Anderson, J. H. Hecht, D. G. Brinkman, R. L. Walterscheid, L. R. Lyons, R. Pfaff, and B. A. Emery, ARIA II neutral flywheel-driven field-aligned currents in the postmidnight sector of the auroral oval: A case study, J. Geophys. Res., 102(A5), 9749-9759, 1997.

Ogasawara, K., K. Asamura, T. Takashima, Y. Saito, and T. Mukai, An energetic electron precipitation observed by the sounding rocket S-31035, Earth Planets Space, 58, this issue, 1155-1163, 2006.

Oya, H., Study on boundary value problems of magneto-active plasma and their applications to space observation, Ph.D. thesis, Kyoto University, Japan, 1966.

Oya, H. and T. Aso, Ionospheric electron temperature measured by a gyroplasma probe, Spece Research IX-North-Holland Publishing Comp., Amsterdam, 287-296, 1969.

Parish, H. F., R. L. Walterscheid, P. W. Jones, and L. R. Lyons, Simulations of the thermospheric response to the diffuse aurora using a three-dimensional high-resolution model, 108(A4), 1140, doi: 10.1029/ 2002JA009610, 2003

Price, G. D., R. W. Smith, and G. Hernandez, Simultaneous measurements of large vertical winds in the upper and lower thermosphere, J. Atmos. Terr. Phys., 57(6), 631-643, 1995.

Rees, M. H. and J. C. G. Walker, Ion and electron heating by auroral electric fields, Ann. Geophys., 24, 193, 1968.

Takahashi, T., H. Oya, H. Fukunishi, Y. Watanabe, and H. Yamagishi, Structure of the auroral ionosphere observed by the antarctic rockets S-310JA-11 and -12, J. Geomag. Geoelectr., 40, 843-857, 1988.

Thiele, B., R. Boström, A. Dumbs, K. U. Grossman, D. Krankowsky, P. Lämmerzahl, G. Marklund, E. Neske, G. Schmidtke, and K. Wilhelm, In situ measurements of heating parameters in the auroral ionosphere, Planet. Space Sci., 29(4), 455-468, 1981.

Wakabayashi, M. and T. Ono, Multi-layer structure of mid-latitude sporadic-E observed during the SEEK-2 campaign, Ann. Geophys., 23, 2347-2355, 2005.

Wakabayashi, M., T. Ono, H. Mori, and P. A. Bernhardt, Electron density and plasma waves in mid-latitude sporadic-E layer observed during the SEEK-2 campaign, Ann. Geophys., 23, 2335-2345, 2005.

M. Wakabayashi (e-mail: waka@stpp1.geophys.tohoku.ac.jp) and T. M.
Ono 